focus on German Studies.

https://journals.uc.edu/index.php/fogs

Issue 28 (2021)

\title{
„Aussterben ist vornehm“ - Eduard von Keyserlings degenerierter Adel im Spiegel der Natur
}

\author{
Marja Kersten \\ Universität Bielefeld
}

Abstract

Der Beitrag untersucht die Darstellung der deutsch-baltischen Adelsgesellschaft in Eduard von Keyserlings

Eræä̆hlungen Schwüle Tage, Harmonie und Am Südhang. Die Analyse zeigt, inwiefern es sich bei Keyserlings Adlige

um das Abbild eines degenerierenden Standes handelt, der nach der Jahrhundertwende stark an Bedeutung verliert. Der Beitrag veranschaulicht, wie Keyserling diesen Niedergang mit Farb-, Pflanzen- und Wettermetaphorik darstellt.

Keywords:

Keyserling - Adel - Degeneration - Jahrhundertwende - Natur

ORCID ID: https://orcid.org/0000-0003-0253-7306

How to cite: Liese, Lea. "Die unverfälschte Gemeinschaft. Authentifikationsstrategien einer exklusiven Geselligkeit bei Achim von Arnim und Clemens Brentano“. focus on German Studies 27: Spielformen des Authentischen, no. 27, 2020, pp. 20 -

43. DOI: $10.34314 /$ FOGS2021.00003 


\section{„Aussterben ist vornehm“ - Eduard von Keyserlings degenerierter Adel im Spiegel der Natur}

\section{Marja Kersten}

Bis zu seinem Tod 1918 verfasste Eduard von Keyserling, Autor aus einem deutsch-baltischen Adelsgeschlecht, zahlreiche Erzählungen, mehrere Romane und Dramen sowie Essays. Ein Großteil seiner Werke thematisiert das Leben adliger Figuren. Heute zählen viele dieser Werke zu den sogenannten Schlossgeschichten. Dies ist nicht verwunderlich, wenn man Keyserlings adlige Abstammung berücksichtigt. Was in der Forschungsliteratur als Adelsenklave bezeichnet wird (Schwalb 11), findet in Keyserlings Werk einen literarischen Höhepunkt. Das adlige Landgut wird zum entscheidenden Handlungsort der Erzählungen. Wie grenzt sich die deutsch-baltische Adelswelt in Keyserlings Erzählungen von der nicht-adligen Außenwelt ab? Nutzt sie neben einer inneren Abgrenzung auf die räumliche Abgrenzung in Form einer Adelsenklave?

In Anbetracht des historischen Kontextes der Jahrhundertwende stellt sich in diesem Beitrag die Frage, wie Keyserling eine Adelsgeneration abbildet, die ahnt, dass sie kontinuierlich an gesellschaftlicher Bedeutung verliert. Spiegelt sich der Niedergang des realhistorischen deutschen bzw. deutsch-baltischen Adels um die Jahrhundertwende in Keyserlings Werk wider? Ausgangspunkt dieses Beitrags ist die These, dass die Darstellung eines degenerierenden Adels in Keyserlings Kurzprosa ersichtlich wird: Der Rückzug in eine Adelsenklave und die damit verbundene Abgrenzung von der nicht-adligen Welt könnte Keyserlings Adligen dazu dienen, die Zeit des Niedergangs anzuhalten, den Prozess der Degeneration zu entschleunigen und eine vermeintliche Sicherheit zu schaffen, die der realhistorische Adel nach der Jahrhundertwende zu verlieren scheint. 
focus on German Studies.

https://journals.uc.edu/index.php/fogs

(ISSN 1076-5697)

Keyserling nutzt verschiedene Motive und Gestaltungsmittel, um das adlige Leben auf deutsch-

Wettermetaphorik. Diese Form der Naturmetaphorik soll die Grundlage für den folgenden Beitrag bilden.

\section{Methodische Rahmung}

Um 1900 rückt in der Literatur das Subjekt, der Mensch als Individuum in den Vordergrund - diese Thematisierung ist nicht neu, ist sie doch ein entscheidender Bestandteil des aufklärerischen Humanismus. Zur Jahrhundertwende wandelt sich der Subjekt-Begriff allerdings; im Interesse steht nun vornehmlich das Negativum des Subjekts - in Form eines Subjektzerfalls (Kimmich und Wilke 70f.). „Um narrative Strukturen zu entwickeln, in denen sich die Erfahrung subjektiver Desintegration adäquat gestalten lässt, greifen Autoren um 1900 auf kurze Prosaformen [...] zurück“ (Kimmich und Willke 71). Auch Keyserling verfasste größtenteils Erzählungen und Novellen. Da auch die Degeneration des Adelsstands als eine Form des Subjektzerfalls gedeutet werden kann, soll der Schwerpunkt in dieser Untersuchung ebenfalls auf Keyserlings Kurzprosa liegen.

In diesem Beitrag werden beispielhaft seine Erzählungen Schwüle Tage (1904), Harmonie (1905) und Am Südhang (1911) betrachtet. Untersucht wird, wie Keyserling die Adligen auf ihren Landgütern darstellt, und vor allem, inwiefern Pflanzen- und Farbmetaphorik zu dieser Darstellung beitragen. Lässt sich ein für die Jahrhundertwende typischer, literarischer Subjektzerfall an Keyserlings Adligen erkennen? Lässt sich in dieser Darstellung eine realhistorische Authentizität erkennen, und wenn ja, hat das überhaupt Relevanz für die Rezeption der Erzählungen?

\section{Farben, Landschaft und Pflanzenmetaphorik}


focus on German Studies.

https://journals.uc.edu/index.php/fogs

focus

(ISSN 1076-5697)

on German Studies

Seit jeher sind Farbgestaltung und die Verwendung von Pflanzenmetaphorik Bestandteile

literarischer Textgestaltung. In der Tradition der deutschsprachigen Literaturgeschichte ist besonders die Pflanzenmetaphorik mit der christlichen Tradition verbunden. „Wer würde, wenn er mit der Tradition einigermaßen vertraut ist, bei Lilie und Rose(nkranz) nicht an die Jungfrau Maria denken?“ (Birkhan 14). Auch in Eduard von Keyserlings literarischem Werk finden sich zahlreiche Farb- und Pflanzenmetaphern, die vor allem Stimmungen und Gefühlszustände der Figuren abbilden (Buchlaub und Wefel 249). Keyserlings Darstellung der Adligen ist häufig verbunden mit der Unterdrückung von Emotionen und persönlichen Sehnsüchten der Figuren. Grund dafür scheint der adelsgesellschaftliche Druck zu sein, dem sich Keyserlings Adlige ausgesetzt sehen. Sabine Buchlaub und Claudia Wefel stellen in ihrer Untersuchung der Landschaftsdarstellung bei Keyserling folgende These auf:

Die Natur dient nicht als Ort der Handlung und auch nicht nur als rasch wechselnder Sinneseindruck, sondern ihre Details werden auf unterschiedlichen Ebenen mit menschlichen Empfindungen und Handlungen in Verbindung gebracht. Natur kann sich bei Keyserling zur Seelenlandschaft erweitern, zu einem Abbild und Echo des menschlichen Gemütszustandes (249).

Inwiefern sich solche Landschafts- und Naturbeschreibungen auf Keyserlings Adlige beziehen lassen, soll in diesem Beitrag dargelegt werden. Ein entscheidender Gesichtspunkt sind die vielen Vergleiche von Frauen mit Blumen sowie die Verbindung einzelner Figuren mit Pflanzen. Die an der häufigsten genannten Blume ist die Lilie, die vornehmlich mit den adligen Mädchen verknüpft ist (Keyserling, "Schwüle Tage" 22). Dieser Vergleich ist naheliegend, da die Lilie für Keuschheit und Unschuld steht (Lauinger 697) - Attribute, die auch den adligen Frauen zugeschrieben werden. Bei der Lilie zeigt sich zudem ein starker realhistorischer Bezug zum Adel, da sie seit Jahrhunderten mit dem Adel in Verbindung gebracht wird - so beispielsweise auf Wappen.

\section{Beispiel 1: Schwüle Tage}


focus on German Studies. https://journals.uc.edu/index.php/fogs (ISSN 1076-5697) focus on German Studies

Schwüle Tage (1904) erzählt vom jungen Adligen Bill von Fernow, der mit seinem Vater Gerd den Sommer auf dem Landgut der Familie verbringt. Bill besteht seine Abiturprüfung nicht und soll auf dem Landgut für eine Nachprüfung lernen. Der junge Mann ist in seine Cousine Gerda Warnow verliebt, die seine Gefühle nicht erwidert. Seine Cousine Ellita verlobt sich auf Wunsch der Familie mit Bills Cousin Went. Bill findet heraus, dass Ellita jedoch eine heimliche Beziehung mit seinem Vater Gerd hatte. Er bemerkt, dass Gerd schwerwiegende psychische Schwierigkeiten hat, die er vehement zu verheimlichen versucht. Bill fühlt sich einsam auf dem Landgut und sucht Trost beim Dienstmädchen Margusch. Kurz nach der Abreise von Gerda und Ellita nimmt sich Gerd das Leben. Interessant ist an dieser Erzählung unter anderem die Dauer des Erzählens, da das Verhältnis von Erzählzeit und erzählter Zeit häufig wechselt. Dadurch entsteht ein beinahe wellenförmiges Zeitgefühl - und passend zum Flirren in der Mittagshitze der Schwülen Tage eine Oszillation des Zeitgefühls.

Nahezu alle Blumen, die im Zusammenhang mit den adligen Figuren stehen, sind Zierpflanzen, die in der Erzählung Schwüle Tage weitestgehend in Blumenbeeten angeordnet sind; so gehen die Adligen Bill und Ellita beispielsweise „zwischen den Lilienbeeten den Weg hinunter“ (Keyserling, “Schwüle Tage” 22). In der strukturierten Anordnung der Pflanzen in Blumenbeeten zeigen sich die Künstlichkeit und das ausgeprägte Ordnungsbewusstsein der Adelsgesellschaft. Die Blumenbeete befinden sich in einem zum Landgut gehörigen Park, durch den die Adligen flanieren. Der geschützte Raum der künstlich angelegten Parkanlage verstärkt den Eindruck einer Adelsenklave, in die sich Keyserlings Adlige zurückziehen (Buchlaub und Wefel 257). Dies zeigt sich auch im Kontrast der Parkgestaltung zum nahegelegenen Wald. Interessant ist, dass die Darstellung der Pflanzen in Schwüle Tage immer mit sensorischer und ästhetischer Wahrnehmung verbunden ist. Dazu zählt zum einen die Verbindung von visueller und olfaktorischer Wahrnehmung und zum anderen die ästhetische Wahrnehmung Bills von Pflanzen und Frauen. Beides steht häufig in Zusammenhang; Bill nimmt zuerst die Landschaft um sich herum wahr und überträgt die 
focus on German Studies. https://journals.uc.edu/index.php/fogs (ISSN 1076-5697)

Wahrnehmung ebendieser dann auf die Wahrnehmung der Frauen:

Angenehm wurde es erst, als die Sonne unterging. Ich spazierte mit den Mädchen langsam an den Blumenbeeten entlang und machte sie lachen. Am Gartenrande blieben wir stehen und sahen über die Felder hin. Rotes Gold zitterte in der Luft. Der Duft von reifem Korn, blühendem Klee wehte herüber. (Keyserling, "Schwüle Tage" 20)

Schwüle Tage bietet zahlreiche Beispiele dafür, wie Zierpflanzen und Blumenarrangements den Drang zur Selbstdarstellung der Adligen veranschaulichen. Gleichzeitig aber zeigen sie Missstände und Konflikte innerhalb der Adelsgesellschaft oder in Bezug auf einzelne Figuren auf. Beispielhaft dafür ist eine kurze, auf den ersten Blick banale Handlung des jungen Adligen Went. Dieser geht einen Weg durch den Park entlang und „köpf $[\mathrm{t}]$ mit seiner Reitgerte die roten Floxblüten“ (Keyserling, "Schwüle Tage” 37). Phloxblüten zählen ebenfalls zu den Zierpflanzen und „symbolisieren Zusammengehörigkeit“ (Lauinger 669). Das Zerschlagen dieses Gewächses wird mit dem gewaltvollen Verb köpfen ausgedrückt; betrachtet man die Zierpflanzen als Sinnbild für die Normen und Sitten des Adels sowie dessen Künstlichkeit, lässt sich diese Szene so interpretieren, dass Went - als Repräsentant der jüngeren Adelsgeneration - die Traditionen und Werte der vorherigen Jahrhunderte zerschlägt, die für ihn nicht mehr vertretbar zu sein scheinen. Auch der Aspekt der Zusammengehörigkeit fügt sich in dieses Bild ein: Innerhalb der Adelsenklave scheint es kein aufrichtiges Gemeinschaftsgefühl zu geben, das sich nicht auf den Adelsstand bezieht. Auch dieses Gefühl zerschlägt Went mit seiner Gerte.

Den Zierpflanzen stehen Nutzpflanzen gegenüber, die mit dem nicht-adligen Personal in Verbindung gebracht werden. So pflückt das nicht-adlige Mädchen Marrie beispielsweise „Rittersporn und Majoran“ (Keyserling, "Schwüle Tage” 35), und Bauernmädchen stehen in den Kohlbeeten, um dort zu arbeiten (Keyserling, "Schwüle Tage” 34). Das ist insofern von Bedeutung, als die Bauern und Angestellten nützlichen, zumeist körperlichen Tätigkeiten nachgehen müssen, um ein Einkommen zu sichern, während 
focus on German Studies. https://journals.uc.edu/index.php/fogs (ISSN 1076-5697) focus on German Studies

für Keyserlings Adlige Folgendes gilt: „Was die zur Schloßwelt Gehörigen gern als „Arbeit“ bezeichnen, sind [...] Tätigkeiten mit Zeitvertreibscharakter, denen man den Anstrich von [...] Nützlichkeit verleiht, um sich [...] zu legitimieren“" (Schulz 132).

Die Verwendung von Pflanzenmetaphorik hängt stark mit der Farbmetaphorik zusammen. „Die Dominanz des Farbkontrastes ist in den Werken Keyserlings augenscheinlich, daneben ,malt ${ }^{\natural}$ er jedoch auch farbenprächtige und bunte Szenerien“ (Buchlaub und Wefel 246), die einen entscheidenden Einfluss auf Stimmung und Atmosphäre haben. So ist es möglich, den Gemütszustand der adligen Figuren mit hellen oder dunklen Farben zu veranschaulichen.

Anfangs sind es vornehmlich helle Farbtöne wie Gelb, Weiß und Rot, die häufig genannt werden. Mit der Verschlechterung des Gemüts- und Gesundheitszustandes von Gerd, die mit der unerfüllten Liebe zu Ellita einhergeht, werden die Farben immer dunkler. Zu Beginn werden Landschaft und Gartenanlage des Landgutes mit Worten wie diesen beschrieben: „Vom Hügel aus sahen wir Warnow vor uns liegen: die Lindenallee, das weiße Haus zwischen den alten Kastanienbäumen“ (Keyserling, "Schwüle Tage” 17) oder „[d]ie Lilien leuchteten weiß aus der Dämmerung“" (Keyserling, "Schwüle Tage” 18). Beides trägt die adlige Selbstdarstellung exteriori; die positive Bedeutung der Linde lässt sich bis auf die biblische Tradition zurückführen (Birkhan 28). Umso drastischer stellt sich der Kontrast von adliger Selbstdarstellung und innerer Gefühlslage der Adligen dar.

Wie der Titel bereits erahnen lässt, sind viele dieser Beschreibungen mit Wärme verbunden, die sich immer stärker zu einer bedrückenden Schwüle entwickelt. Zunächst scheint eine nahezu idyllische Stimmung auf dem Landgut zu herrschen - im Verlauf der Handlung tauchen jedoch zunehmend düstere Beschreibungen sowie dunkle Farben auf. In der zweiten Hälfte der Erzählung finden sich viele Beispiele wie: „Es war finster geworden“ (Keyserling, “Schwüle Tage” 56), „schwarzer Wolkenstreifen“ (Keyserling, “Schwüle Tage” 54), „Die Nacht war schwarz. Einige welke Blätter raschelten schon auf dem Wege“ 
focus on German Studies.

https://journals.uc.edu/index.php/fogs

(ISSN 1076-5697) focus

on German Studies

(Keyserling, “Schwüle Tage” 80) oder „Ein feiner Regen begann in den Weiden und im Schilf zu flüstern“

(Keyserling, "Schwüle Tage” 56). Auch die Beschreibung des Leichnams von Gerd ist düster, gleichzeitig aber mit Schönheit - beinahe schon Erhabenheit - verbunden. Diese Verbindung erzeugt eine mystische Atmosphäre:

Das fahle Gesicht mit der hohen Stirn, die im Mondlicht matt glänzte, lächelte noch immer sein verhaltenes, schiefes Lächeln, die Augen waren geschlossen, die langen Wimpern legten dunkle Schattenränder um die Lider. Aber wenn der Mond sich verfinsterte, schien es mir, als bewegten sich die Umrisse der Gestalt, ich fühlte wieder, dass er mich ansah (Keyserling, "Schwüle Tage” 100).

Die Kontrastierung von Licht und Dunkelheit findet sich kontinuierlich in Keyserlings literarischem Werk: „Die Keyserling'schen Landschaftsbilder sind gekennzeichnet durch das von Jahres- und Tageszeiten abhängige Licht; vertraute Landschaften und Gärten werden so zu variationsreichen Stimmungsfaktoren des Augenblicks“ (Buchlaub und Wefel 243). Dass Bill den Leichnam seines Vaters findet, muss folglich im Dunkeln geschehen; das Licht des Mondes, das die Dunkelheit erhellt, schafft eine Art Feierlichkeit, die das anmutig wirkende Gesicht des Leichnams betont. Dieser Licht-und-Schatten-Kontrast lässt sich auf zahlreiche weitere Textstellen in den Erzählungen Keyserlings übertragen und verdeutlicht einmal mehr die innere Zerrissenheit der adligen Figuren, die sich zwischen Tradition und Aufbruch befinden.

Doch nicht nur der sich verschlechternde Zustand Gerds, sondern auch Bills eigene Unzufriedenheit sowie das schwierige Verhältnis zu seinem Vater spiegeln sich in der Farbauswahl wider. Besonders zeigt sich dies nach Bills Gespräch mit Ellita, wenn er seine Wahrnehmung folgendermaßen beschreibt: „Die Nacht war schwül. Regungslos und schwarz standen die Bäume im Garten. In der Ferne donnerte es“ (Keyserling, “Schwüle Tage" 54). Hier deutet sich das Gewitter an, welches kurz darauf ausbrechen wird.

Bereits der Titel der Erzählung lässt vermuten, dass sich eine Problematik anbahnt beziehungsweise eine große Anspannung aufgebaut wird, die sich möglicherweise in einem Gewitter lösen wird - allerdings ist das 
focus on German Studies. https://journals.uc.edu/index.php/fogs (ISSN 1076-5697) focus

on German Studies

Gewitter in diesem Fall eine Katastrophe und keine Auflösung der Konflikte innerhalb der Adelsgesellschaft. Betrachtet man die drückende Schwüle allerdings als Beklemmung des Adels, könnte der Suizid zumindest aus Gerds Perspektive als eine Form der Befreiung gesehen werden, die - im übertragenden Sinn - die Schwüle mit einem Wolkenbruch auflöst.

Die Schwüle, die nicht nur im Titel, sondern auch an mehreren Stellen innerhalb der Erzählung vorkommt, scheint wie eine Unheilsverkündung über den Figuren zu hängen. Gleichzeitig kann die Schwüle auch so gelesen werden, dass bis zum Gewitter - das heißt: auch im übertragenen Sinne einer Entladung eine Art Sicherheit für die Figuren fühlbar ist, da sich in diesem Zustand der Schwebe nichts zu verändern scheint: „In Warnow war alles, wie es sein mußte. Ein jedes Zimmer hatte noch seinen gewohnten Geruch“ (Keyserling, "Schwüle Tage” 17). Vor allem Bill scheint in der Adelsenklave des Landgutes Beständigkeit und vermeintliche Sicherheit zu sehen, die ihm in seiner Innenwelt und auch in seinem gesellschaftlichen Leben fehlt. Solcher Stillstand, den man schnell mit Hitze und Schwüle in Verbindung bringt, findet sich immer wieder in der Erzählung. So beispielsweise beim Blick aus dem Fenster des Landguts: „Der Kies flimmerte. Alle standen sie unbeweglich in der Glut, müßig und faul unter dem schläfrigen Summen, das durch die Luft zog“ (Keyserling, "Schwüle Tage” 15). In mehreren Textpassagen sinniert Bill über das Gefühl, das die Schwüle in ihm auslöst. Dabei scheint es ihm besonders erwähnenswert zu sein, wenn sich etwas nicht verändert. So auch, als Bill auf den Treppenstufen des Landgutes sitzt und wieder in Gedanken vertieft ist:

Hier lag wieder alles vor mir und dort drinnen saß die in sich zusammengekrümmte Gestalt mit den tragisch über das Gesicht gespreizten Händen. Etwas Unbegreifliches war in der Verschwiegenheit der Mittagsstunde entstanden (Keyserling, "Schwüle Tage" 40). Das Unbegreifliche, das Bill hier zu reflektieren versucht, ist, dass er seinen sonst so beherrschten Vater beim Weinen gesehen hat, nachdem dieser von der Verlobung Ellitas mit Went erfahren hat (Keyserling, "Schwüle Tage” 37ff.). Doch wie bei einer 
focus on German Studies.

https://journals.uc.edu/index.php/fogs

(ISSN 1076-5697)

meteorologischen Schwüle folgt auf das Ausharren in der Hitze auch in der Erzählung ein Gewitter, das die Spannung nur indirekt löst, in diesem Fall aber gleichzeitig eine große Tragik mit sich bringt.

\section{Beispiel 2: Harmonie}

Protagonist der Erzählung Harmonie (1905) ist der junge Adlige Felix von Bassenow, der nach einem Aufenthalt in Italien auf das heimatliche Landgut zurückkehrt. Dort wartet seine Ehefrau Annemarie, die nach dem Tod ihres gemeinsamen Kindes psychisch so labil ist, dass sie in seiner Abwesenheit in ein Sanatorium eingewiesen wurde. Auch Felix kann mit der schwierigen Lebenssituation nicht umgehen und entschließt sich daher zu ebendieser Italienreise. Nach seiner Rückkehr ist das Verhältnis zwischen den Ehepartnern gestört. Felix versucht, sich Annemarie anzunähern, findet aber keinen Zugang mehr zu ihr. Doch nicht nur zu seiner Ehefrau, sondern auch zu den anderen Bewohnern des Landguts kann Felix kein enges Verhältnis mehr aufbauen. In Felix’ Abwesenheit kommt Annemarie dem deutlich älteren Thilo näher, der Gefühle für Annemarie aufbaut. Felix fühlt sich von Thilo in seiner Rolle als Annemaries Ehemann bedroht. Er sucht Rat bei Frau von Malten, einer Freundin Annemaries, die ihm die Schuld an dem distanzierten Verhältnis gibt, da Felix seine Frau in ihrer Trauer verlassen hat. Frau von Maltens Pflegetochter Mila zeigt Interesse an Felix, der sich auf ein sexuelles Verhältnis mit ihr einlässt, ihre Gefühle jedoch nicht erwidert. Da sich Felix weiterhin von Thilo gestört fühlt, stellt er diesen zur Rede; Thilo streitet ab, amouröse Gefühle für Annemarie zu hegen. Trotzdem entschließt sich Thilo dazu, das Landgut zu verlassen. Felix erkennt, dass sich ein inniges Verhältnis zwischen seiner Ehefrau und Thilo entwickelt hat. Annemarie kann mit Thilos Abreise nicht umgehen und beschließt, sich das Leben durch Ertrinken zu nehmen. Felix versucht, sie zu retten, doch Annemarie stirbt. Felix zeigt sich als innerlich zerrissener Adliger, der mit dem Verlassen der Adelsenklave scheinbar eine Katastrophe ausgelöst hat, die er nicht 
focus on German Studies.

https://journals.uc.edu/index.php/fogs

(ISSN 1076-5697) focus

on German Studies

aufhalten kann. In der Fokussierung auf sich selbst sieht er seine adelsgesellschaftliche Position bedroht und erkennt die Gefahr von Annemaries psychischer Verfassung nicht. Seine Rückkehr und der Versuch, sich wieder in sein altes Leben einzufügen, erinnern an „das alte literarische Motiv des odysseischen Heimkehrers“ (Markewitz 27).

Stärker noch als in Schwüle Tage zeigt sich in Harmonie die enklavische Abgrenzung der Adelsgesellschaft vom nicht-adligen Außenraum. Die Trennung führt so weit, dass Felix, der die Adelsenklave verlassen hat und nun wieder zurückkommt, kein akzeptiertes Mitglied der Adelsgesellschaft ist, obwohl er bemüht ist, sich zu assimilieren (Keyserling, "Harmonie" 110). Innerhalb der Adelsenklave ist Individualität nicht gern gesehen. Die Gespräche bleiben recht oberflächlich, und im Vordergrund steht immer das Bedürfnis, sich von Nicht-Adligen abzugrenzen, etwas Besonderes zu sein. Felix reflektiert über seine Ehefrau:

Auch von ihm, ihrem Mann, konnte Annemarie nur eine Auslese gebrauchen, sie sah das, was ihr an ihm gefiel, das andere wies sie ab mit dem leichten, ein wenig grausamen Zucken der Lippen, das er fürchtete. Gott!, er hatte sich oft höllisch zusammennehmen müssen, um so zu sein, wie sie ihn sah (Keyserling, “Harmonie" 107).

An Felix - und auch an die anderen adligen Schlossbewohner - wird also der Anspruch gestellt, etwas Besonderes zu sein, obwohl ihre Sitten und Traditionen die individuelle Besonderheit unterdrücken. Damit steht die Außenwirkung ganz augenscheinlich im Vordergrund.

Besonders auffällig wird die Darstellung der Adelsgesellschaft im Kontrast zu der des Bauerntums. Trotz der heterodiegetischen Erzählinstanz stehen die adligen Figuren im Zentrum der Erzählung; ihre Wahrnehmung der Bauern wird trotzdem deutlich. Das Bauerntum wird als ungehobelt und im Umgang mit ihren Tieren als brutal dargestellt (Keyserling, "Harmonie" 113f.). Die Adligen - in diesem Fall hauptsächlich die männlichen Figuren - scheinen das Bedürfnis zu haben, sich vor den unteren Schichten 
focus on German Studies. https://journals.uc.edu/index.php/fogs (ISSN 1076-5697) focus on German Studies

zu profilieren (Keyserling, "Harmonie” 106). Keyserlings Adelsgesellschaft scheint in dieser Erzählung keineswegs eine harmonische zu sein. „Sogar solche vordergründig sanften Frauen wie [...] Annemarie demonstrieren eine nahezu drastische Erbarmungslosigkeit, sobald sie mit Un-Ästhetischem konfrontiert werden“ (Schulz 42) und der äußere Schein ihrer Adelsenklave nicht aufrechterhalten werden kann.

Entscheidend an Landschaftsgestaltung und Pflanzenmetaphorik ist, „daß die Schloßgesellschaft in „Harmonie“ kaum noch ein elementares Verhältnis zur Natur hat“ (Buchlaub und Wefel 245). Damit ist vor allem die Gestaltung der Parkanlagen verbunden: Die Künstlichkeit der kultivierten Natur wird betont durch die Gegenüberstellung der Schlosslandschaft und ,großen Waldgebieten, die die Schloßbezirke umgeben, räumlich abgetrennt durch die Parkgitter, die die Grenzlinien zwischen beiden Bereichen bilden“ (Buchlaub und Wefel 256). Zur „Verkünstlichung der Natur“ (Weinhold 77) kommt außerdem die fingierte Idylle des Landlebens hinzu. Die adligen Figuren beobachten beispielsweise Bauern bei ihrer landwirtschaftlichen Arbeit (Keyserling, "Harmonie" 114), bleiben aber nur Beobachter oder Auftragsgeber. So spricht Onkel Thilo von „einem Durchhau in den Parkbäumen, der sich gut machen würde“ (Keyserling, "Harmonie" 137). Er plant demgemäß einen weiteren Eingriff in die Natur, die in der direkten Umgebung des Schlosses per se schon künstlich ist.

Auch der Wald wird von den Adligen nicht für zweckmäßige, sondern nur für der Unterhaltung dienliche Tätigkeiten genutzt: „Ritualisierte Veranstaltungen wie Jagdausflüge, Picknicks und Kaleschenausfahrten unterliegen einer sorgfältigen Planung und werden nach höfischem Zeremoniell absolviert“ (Buchlaub und Wefel 256). Ergo ist keine Bindung zur ursprünglichen Natur mehr vorhanden. Künstlichkeit findet sich auch auf sprachlicher Ebene: „Im Osten hing ein weißer Lichtstreif am Horizont. [...] Nachtfalter streichelten mit kühlen Sammetflügeln seine Wangen. Sehr hoch über sich hörte er schon die Morgenschnepfen quarren“ (Keyserling, "Harmonie” 134). Die Sprache ist hier besonders künstlich ausstaffiert und ähnelt damit stark dem äußeren Schein, den die Adligen zu wahren versuchen. 
focus on German Studies. https://journals.uc.edu/index.php/fogs (ISSN 1076-5697) focus on German Studies

In der Gestaltung der Parkanlagen finden sich viele Motive des Jugendstils, die Azzone Zweifel herausgestellt hat: ein ,geschlossener Garten, einsamer Park, Teich als Ort des Todes, Schwäne, Fliederlauben, schlingende Wasserpflanzen und die mit Wasser verbundenen Mythen von Narziß und Ophelia“ (Schulz 22). Das Ophelia-Motiv ist hier eine naheliegende Assoziation. Damit wird der Teich zum Ort des Todes. Besonders auffällig ist, dass die Todesmotivik mit der weiblichen Schönheit und der der Natur verknüpft wird. Neben der Verbindung mit der Todesmotivik, wird Annemarie mit Symbolen der Unschuld beschrieben. Das Unschuldsmotiv findet sich bei ihr vor allem in der Verwendung von Pflanzenund Farbmetaphoriken. Thilo und Annemarie bewerfen sich beispielsweise spielerisch mit Blüten und nehmen - wie Thilo es bezeichnet - „Blütenbäder“ (Keyserling, “Harmonie” 123), damit sie sich besser fühlt. Zu einer feierlichen Angelegenheit trägt Annemarie ein „teerosenfarbenes, leichtes Seidenkleid“ (Keyserling, "Harmonie" 116) - ihre Zartheit wird erneut betont. Eine gewichtige Rolle nimmt der Flieder ein, der an mehreren Stellen beschrieben wird: „Der Flieder umgab das Haus wie mit einem Wall von weiß und blass violetten Musselinen“" (Keyserling, "Harmonie” 139). In seiner Vision von Annemaries Tod sieht Felix sie mit einem Fliederzweig in der Hand, den er bei seinem realen Rettungsversuch auch auf der Teichoberfläche schwimmen sieht (Keyserling, "Harmonie” 143). Flieder steht in der Pflanzensymbolik für tiefe Zuneigung und Liebe (Lauinger 668). Das ist insofern eine treffende Metapher, als Felix während der Vision seine Liebe zu Annemarie spürt, die ihm zuvor fremd vorgekommen ist.

Doch nicht nur Annemarie, sondern die gesamte Adelswelt wird mit hellen Farben - vor allem mit Weiß assoziiert. Das Mondlicht erleuchtet die Parkanlage in strahlendem Weiß (Keyserling, "Harmonie” 118) und adlige Frauen tragen helle Kleidung (Keyserling, "Harmonie" 116). Doch die Helligkeit wandelt sich bis zur Düsternis nächtlicher Träume: „Draußen rauschte ein starker Frühlingsregen nieder. Dieses Rauschen sprach in Felix' Träume hinein, füllte sie mit einem weißen, blanken Niederrinnen, das kühl nach Waldblumen duftete“ (Keyserling, "Harmonie” 111). Wie schon in Schwüle Tage, findet sich auch 
focus on German Studies. https://journals.uc.edu/index.php/fogs (ISSN 1076-5697) focus on German Studies

in Harmonie eine auffällige Anzahl olfaktorischer Eindrücke. So beispielsweise an folgenden Textstellen: „Im

Garten blühte der Faulbaum. Sein gewaltsamer Duft benahm fast den Atem“ (Keyserling, "Harmonie” 106), „Der Frühling duftete hier schwüler“ (Keyserling, "Harmonie” 107) oder die Wahrnehmung von Veilchenduft (Keyserling, “Harmonie” 119). Veilchen gelten als „Symbol für unschuldige Liebe und Zartheit“ (Lauinger 673) und spiegeln damit wider, was den adligen Frauen zugeschrieben wird. Diese Zuschreibung findet sich bereits „,in der Frauenlob-Schule [, in der] die Angebetete als [...] ,Veilchengarten“ angeredet wird“ (Birkhan 12).

Die hellen, blassen Farben der adligen Welt stehen im Kontrast zur bäuerlichen Welt, die - genau wie auch der nahegelegene Waldbezirk - mit kräftigen Farben assoziiert wird. So wird der Wald folgendermaßen beschrieben: „Die Droschke fuhr durch den dunkeln Wald, wie zwischen hohen, schwarzen Wänden hin“ (Keyserling, "Harmonie” 126). Dadurch entsteht eine interessante Umkehrung: Obwohl die Adelswelt mit hellen Farben verbunden ist und die bäuerliche Welt mit kräftigeren Farben, ist es die Adelswelt, durch die sich die Todesmotivik zieht. Ergo zeigt sich auch in der Farbmetaphorik der äußere Schein, der gewahrt werden muss, um seinesgleichen zu beeindrucken, während die bäuerliche Welt mit ihren kräftigen Farben Lebendigkeit verkörpert.

Die Adelsenklave scheint einen enormen Einfluss auf die Persönlichkeit der Adligen zu haben; auch Felix erkennt dies bald: „Hier war man doch ein anderer als da draußen“ (Keyserling, “Harmonie” 111). Dieser Gedanke bekommt beinahe etwas Magisches, wenn man folgende Aussage von Felix betrachtet: „Wir leben in einem verzauberten Schloss“ (Keyserling, "Harmonie” 130). Felix nimmt die Adelsenklave demnach als verzaubert war. Seine Gefühle geraten durcheinander; er fühlt sich in seinem Leben gefangen. „Er war mit sich, mit Annemarie unzufrieden. Sie [...] schien ihm fremder und ferner denn je. Und doch war der Wunsch, ganz zu ihr zu gehören, gerade so quälend stark“ (Keyserling, "Harmonie” 133). Ulrike Weinhold fasst das Problem in dieser Ehe wie folgt zusammen: 
focus on German Studies.

https://journals.uc.edu/index.php/fogs

(ISSN 1076-5697) focus

on German Studies

Einer sieht im anderen nur sich; der andere ist bloß Zweck seiner Bedürfnisse, weil sich das solitäre

Ich nur auf sich selbst bezieht und von daher ein unbegrenztes Machtstreben entwickelt, das es durch Negation des Du realisieren will (79).

Egozentrismus ist eine der zentralen Problematiken der Erzählung. Dazu zählt auch „[d]ie Scheu vor dem Mitleid, [und] seine Gleichsetzung mit Krankheit“ (Schulz 40). Einen Grund dafür sieht Angela Schulz darin, dass „Mitleid vom narzißtischen Ich eine ernsthaftere Auseinandersetzung mit einem leidenden Du verlangt und seine Idylle im schönen Schein empfindlich stört“ (ebd.). Auf die Harmonie, die der Titel der Erzählung verspricht, wartet man vergeblich.

Interessant ist außerdem, dass die Adligen in Harmonie scheinbar keine Nachfolger haben. Der einzige erwähnte Nachwuchs ist das verstorbene Kind von Annemarie und Felix. Und auch „Onkel Thilo heiratete nicht, um der letzte Reichsgraf zu Elmt zu sein“ (Keyserling, "Harmonie” 113). Die Degeneration zeigt sich also neben dem Scheitern der Kommunikation, dem Egozentrismus und den zahlreichen Todesmotiven auch in der fehlenden Fortsetzung des Adelsgeschlechts. Keyserlings Adlige gehen sogar noch einen Schritt weiter und sagen: „Aussterben ist vornehm“ (ebd.).

\section{Beispiel 3: Am Südhang}

Ähnlich wie in Schwüle Tage, verbringt auch der Protagonist in Am Südhang den Sommer auf dem familialen Landgut. Karl Erdmann von West-Wallbaum, adliger Leutnant, besucht seine Familie auf ebenjenem. Ebenfalls Besucherin auf dem Adelssitz ist Daniela von Bardow, für die Karl seit seiner Jugend Gefühle hegt. Das gilt auch für den Hauslehrer Aristides Dorn, der auch Interesse an Daniela zeigt. 
focus on German Studies. https://journals.uc.edu/index.php/fogs (ISSN 1076-5697) focus on German Studies

Karls eigentlich positiv erwarteter Aufenthalt wird dadurch getrübt, dass er wegen eines Streits mit einem anderen Mann an einem Duell teilnehmen soll, das jedoch einen friedlichen Ausgang nimmt, da beide Duellanten absichtlich danebenschießen. Als Aristides erfährt, dass sich Daniela und Karl körperlich angenähert haben, verliert er all seine Hoffnung und erschießt sich. Aristides’ Suizid führt dazu, dass sich Daniela ihre Gefühle für ihn eingesteht und diese offen bekundet. Die Erzählung endet mit der Beisetzung Aristides' und der Abreise Danielas.

Auch in Am Südhang sind Farb- und Pflanzenmetaphorik entscheidend für die Darstellung der Adelsgesellschaft. Ein Unterschied zu den anderen beiden Erzählungen ist, dass bereits zu Beginn der Erzählung „eine Reflexion über das Farbthema eingeflochten ist, die von Karl Erdmann selbst vorgenommen wird“ (Markewitz 32). Seine Gedanken an den bevorstehenden Aufenthalt auf dem Landgut sind geprägt von Wärme:

[A]llein die Tatsache, dass es zu den Ereignissen dieses Sommers gehören würde, gab dem Bilde dieses Sommers, gab der Gestalt Karl Erdmanns doch ein eigenes, ein wenig Licht. So störte denn nichts seine Freude (Keyserling, “Am Südhang” 395f.).

Die Künstlichkeit der Parkanlage des Landgutes scheint von noch größerer Bedeutung zu sein, wenn man das Theatermotiv in diese Überlegung einbezieht: Die künstlichen Gärten werden zur Kulisse des Adels-Schauspiels. Wie in den anderen beiden Erzählungen ist auch in dieser Erzählung die Schwüle von großer Relevanz. Alle visuellen und olfaktorischen Wahrnehmungen münden letztendlich in Schwüle. Diese symbolisiert hier die sich aufladende Spannung zwischen Karl Erdmann und Aristides Dorn sowie deren wachsendes Verlangen nach Daniela, das unerwidert bleibt (Weinhold 75). Die Schwüle - „um 1900 ein besonders oft gebrauchtes Bild für die nervliche Anspannung des modernen Daseins und des gleichzeitigen Wartens auf das große befreiende Ereignis“ (Schneider 156) - bleibt allerdings eine Schwüle und führt nicht zur Auflösung der Spannung. Keyserling wählt mit der Schwüle ein ausgesprochen passendes Beispiel für 
focus on German Studies.

https://journals.uc.edu/index.php/fogs

(ISSN 1076-5697) focus

on German Studies

die Stimmung innerhalb der Adelsenklave: Das bedrückende Gefühl, das die Schwüle bei den Adligen auslöst, geht nicht von einer anderen Figur aus, sondern von der gesamten Atmosphäre innerhalb der Enklave. Auch die Zwänge der adligen Konventionen gehen hier von keiner einzelnen Figur, sondern von der Adelsgesellschaft aus. In beiden Fällen ist es folglich eine Beklemmung, die von außen in den Innenraum eindringt.

Die Farbmetaphorik ist in Am Südhang besonders stark. Die Welt der Adligen ist hier erneut mit hellen Farben - vor allem mit Weiß - verbunden. Das Landgut ist umgeben von weißen Lilien (Keyserling, “Am Südhang” 400), die besonders mit den weiblichen Figuren verknüpft sind: „Beide verkörpern Dekorativität, Erlesenheit bis zur Überzüchtung, Zartheit, Noblesse“ (Schulz 27). Folgt man dieser Lesart, lässt sich eine erotische Komponente in der Pflanzenmetaphorik erkennen. Als Grundlage für diese Überlegung dient folgende Textstelle über Karl nach einer Begegnung mit Daniela: „Dann wartete er [Karl] noch so erregt, dass er mit seinen heißen Händen in die kühlen Lilien hineingriff“ (Keyserling, “Am Südhang” 421). Dieser Kontrast aus Hitze und Kälte betont, dass Karl sexuelles Verlangen nach Daniela spürt, und Daniela, die kühle Lilie, kein Interesse an ihm zeigt.

Obwohl die adligen Figuren kein Verhältnis zur ursprünglichen Natur mehr haben und ihnen der Wald „groß und schwarz“ (Keyserling, “Am Südhang” 445) vorkommt, scheint die sinnliche Wahrnehmung bestimmter Pflanzen Emotionen bei Karl auszulösen. So möchte er vor einem Duell noch einmal den Geruch von Lilien riechen (Keyserling, "Am Südhang” 436) - hier findet sich ein Rückgriff auf das bereits erwähnte erotische Motiv in Bezug auf Daniela. Allerdings ist nun der Unterschied, dass Daniela seinem Verlangen kurz darauf nachgibt und sie nicht mehr die küble Lilie ist. Eine Ausnahme ist Karls Mutter, die ein anderes, weniger gekünsteltes Verhältnis zur Natur hat: Es bereitet ihr Freude, im Garten zu sein und Löwenzahn zu pflücken (Keyserling, “Am Südhang” 432). Doch auch Karls Mutter bleibt in der kultivierten Natur, sucht aber dennoch bewusst nach Augenblicken im Freien. 
focus on German Studies. https://journals.uc.edu/index.php/fogs (ISSN 1076-5697) focus on German Studies

Ein erster Verweis auf die Adelsenklave wird ersichtlich, wenn man die kontrastive Farbgebung aller Orte betrachtet. Der Wald wird sehr dunkel beschrieben, die Farbgestaltung im angrenzenden Dorf ist eher rötlich - also kräftige, warme Farben (Keyserling, “Am Südhang” 441) -, während die Farbgestaltung zum Landgut hin immer heller wird (Keyserling, “Am Südhang” 400). Man kann folglich sagen, dass das Bild des Adels und damit im übertragenen Sinne auch der Adel per se verblasst und immer stärker an Bedeutung für das gesamtgesellschaftliche Leben verliert. Die Adelsenklave, deren Scheitern sich im Verblassen der Farben andeutet, wird in Am Südhang von Karl auch als ebensolche wahrgenommen. Bei seiner Ankunft kommt er sich „wie ein fremder Besuch vor“ (Keyserling, “Am Südhang” 398), der in dem Moment noch nicht wieder Teil der Adelsgemeinschaft geworden ist. Karls Wahrnehmung der Enklave zeigt sich an einer Textstelle ganz besonders deutlich:

Was tat man denn überhaupt hier in dieser Zeit, wenn - wenn man nicht bei Daniela war? [...] Gut, das war es also, was man hier tat. Man sitzt auf einer schattigen Bank, sieht einen roten Sonnenschirm durch all das Grün fahren und denkt an nichts. Nur dass, wenn man von da draußen kommt, so was immer wieder gelernt werden will (Keyserling, “Am Südhang” 403).

Das Gefühl, in einer Enklave zu leben, die nicht zur restlichen Welt gehört, findet in Karls Wahrnehmung eine bislang nicht erreichte Klimax innerhalb der drei Erzählungen: „Entsprechend der räumlichen Enklave ist auch die Zeit einem Kontinuum konkret erfüllter Situationen enthoben und stellt sich als eine leere Wiederholung von Tag und Nacht [...] dar, die als subjektive Stimmungszustände verfließen“ (Weinhold 76). Wie in Schwüle Tage und Harmonie ist die Adelsenklave hier ebenfalls ein entscheidender Bestandteil der Degeneration der dargestellten Adelsgesellschaft.

Aristides macht Karl darauf aufmerksam, dass die Wahrung des äußeren Scheins, Künstlichkeit und Sorglosigkeit nicht die Aspekte sind, die das Leben ausmachen. Er kann in dieser Umgebung nicht glücklich sein und das scheinhafte Schauspiel der Adligen offenbar nicht mehr ertragen (Keyserling, “Am 
focus on German Studies. https://journals.uc.edu/index.php/fogs (ISSN 1076-5697) focus on German Studies

Südhang” 412). Danielas kokettierendes Verhalten trägt zu Karls Wahrnehmung der Adelsgesellschaft bei, da sie die Privilegien der Adelsgesellschaft, aber ebenso die damit verbundene Künstlichkeit repräsentiert, die negativ konnotiert ist.

In jeder der drei Erzählungen kommt es zu einem Suizid; in diesem Fall erschießt sich Aristides unter einem Ahornbaum, da er die unerwiderte Liebe zu Daniela und das scheinhafte Leben in der Adelsenklave nicht länger erträgt (Keyserling, "Am Südhang” 456). Durch die Auswahl des Ortes in $A m$ Südhang entsteht eine Kombination aus den Suiziden in Schwüle Tage und Harmonie: Gerds Leichnam wird unter einem Baum gefunden, Annemarie nimmt sich in einem Teich das Leben und Aristides erschießt sich unter einem Baum, der an einem Teich steht. Hier zeigt sich also eine Steigerung in der Degeneration des Adels. Aristides’ Suizid ist ein erneuter Kontrast zur Künstlichkeit und Unaufrichtigkeit der Adligen. Wichtig ist außerdem, dass es sich bei diesem Suizid nicht um den eines Adligen handelt: Dies legt den Gedanken nahe, dass die Standesunterschiede mit dem Tod aufgehoben werden. Das würde bedeuten, dass die besondere Stellung der Adelsgesellschaft nichtig wird. Die adligen Figuren scheinen die Augen vor ihrer verblassenden gesellschaftlichen Bedeutung zu verschließen und müssen so letztlich scheitern.

Auffällig ist, dass die einzige Figur, die am Schluss von $A m$ Südhang Einsicht zeigt, Karls jüngere Schwester Oda ist. Auch in dieser Erzählung ist es folglich die jüngere Adelsgeneration, die gegenüber einer Umstrukturierung der Adelsgesellschaft nicht gänzlich abgeneigt ist. Erwähnenswert ist an dieser Stelle, dass Oda einen Pflanzenvergleich nutzt, um ihre Bedenken zu veranschaulichen: „Ein Kummer hier bei uns vergeht nicht, es ist hier zu geschützt, er gedeiht hier zu gut, wie alles, wie die dicken Rosen und die großen gelben Pflaumen“ (Keyserling, "Am Südhang” 462). Auf diese Weise entsteht eine motivische Leitlinie in Form der Pflanzenmetaphorik, die sich durch die gesamte Erzählung zieht. Die Fäulnis einer Frucht „,ist ein deutliches Décadencemotiv, es bringt die adelige Lebensform in ein organologisches Bild von Blüte und Verfall“" (Schneider 154). Oda macht Karl darauf aufmerksam, dass das abgeschiedene Leben auf dem 
focus on German Studies.

https://journals.uc.edu/index.php/fogs

(ISSN 1076-5697) focus

on German Studies

Landgut die positiven Aspekte zwar bestärken kann, gleichzeitig aber die mentalen Schwierigkeiten durch das Fehlen äußerer Einflüsse verschlimmert. Die Erzählung endet also mit der Einsicht, dass der Adel langsam, aber stetig degeneriert - mit dem Bewusstsein, dass er Schönheit beinhaltet, aber im

Abgeschottetsein von der restlichen Welt und seinem stetigen Verblassen nicht mehr bestehen kann.

\section{Pflanzen und Farbmetaphorik als Abbild des degenerierten Adels?}

Eduard von Keyserling greift in seiner Darstellung realhistorische Aspekte auf, ohne historische Umstände konkret zu benennen. Auch die Idee der Adelsenklave ist dem realhistorischen Adel nicht fern, wie Historiker Eckart Conze beschreibt:

Auf seinen Gütern kultivierte der Adel eine ,heile Welt': ländliches Leben und Wirtschaften, [...] ideologisch überhöht, patriarchalische Herrschaftsstrukturen und eine exklusive Geselligkeit repräsentierten im kleinen die Welt und die Gesellschaft, deren Verlust im großen der Adel beklagte und den er rückgängig zu machen hoffte (383).

Der Rückzug in eine Enklave ist für Keyserlings adlige Figuren keine gelingende Lösung, um die Degeneration des Adels aufzuhalten. Technische Neuerungen und soziale Veränderungen finden ihren Weg nicht bis in die Adelsenklave hinein. Auf den ersten Blick wirkt es wie ein Ausweg, sich von der Außenwelt abzugrenzen, um nichts Unerwünschtes in die eigene Umgebung hineinzulassen. Für den realhistorischen Adel war die Abgrenzung von anderen Gesellschaftsgruppen von großer Bedeutung und Teil ihres Standesbewusstseins - und auch bei Keyserling leben die Adligen in ihrer Enklave ein eigenes Tempo, das sehr viel langsamer zu sein scheint als außerhalb der Landgüter (Markewitz, "Natur, Kultur, Moderne” 253). Die Adelsenklave wird für Keyserlings Adlige zu einem Rückzugsort, an dem sich ihre Erwartungen allerdings nicht erfüllen können. Ein Großteil der „Adelsmitglieder scheint grundsätzlich jede Transformation, insbesondere jede Innovation abzulehnen: Alles soll so bleiben wie es zur Zeit der jeweils 
focus on German Studies. https://journals.uc.edu/index.php/fogs (ISSN 1076-5697) focus on German Studies

älteren Generation war“ (Wünsch 204). Das bedeutet zwar, dass ein Stillstand innerhalb der Enklave möglich ist, der den Bewohnern das Gefühl einer Entschleunigung gibt, doch gleichzeitig bedeutet der Stillstand auch, dass es keinen Fortschritt und damit kein Fortbestehen geben kann.

In den ausgewählten Erzählungen ist eine klare Komparation zu erkennen: Obwohl sich Gerd in Schwüle Tage das Leben nimmt, bleibt am Schluss für den autodiegetischen Erzähler Bill die Hoffnung auf eine Veränderung seines persönlichen Lebens, die sich in der Aufklarung der Farben und des Wetters zeigt. Der Suizid in Harmonie ist der Schluss der Erzählung und lässt so keinen Ausblick auf eine positive Zukunft zu. Annemaries Suizid, der durch mehrere Faktoren ausgelöst wird, lässt sich mit der Kinderlosigkeit in Verbindung bringen: Sie ist die einzige der weiblichen Hauptfiguren, die bereits ein Kind zur Welt gebracht hat - allerdings ist dieses bereits kurz nach der Geburt verstorben. Mit ihrem Suizid hat Annemarie nicht mehr die Möglichkeit, ein weiteres Kind zu gebären, das ihre Adelslinie fortsetzen könnte; auch an dieser Stelle zeigt sich die Degeneration des Adelsgeschlechts. Am Südhang lässt schließlich keinen Zweifel mehr zu, dass es sich bei Keyserlings Adel um einen degenerierten Adel handelt. Während Bill noch hoffnungsvoll ist und Felix Zweifel an seinem adligen Leben hegt, ist es in $A m$ Südhang eindeutig, dass der Adel in dieser Form nicht weiter bestehen kann. Der einzige Hoffnungsschimmer geht von Karls jüngerer Schwester Oda aus, die die Problematik erkennt und das Leben innerhalb der Adelsenklave mit einer faulenden Frucht vergleicht. Dass es sich bei jedem der drei Tode um einen Suizid handelt, deutet darauf hin, dass die Degeneration des Adels nicht von außen kommt, sondern dass sich der Adel von innen heraus selbst zerstört. Ein Grund dafür könnte das Festhalten an überholten Traditionen sein, welches die adligen Figuren daran hindert, sich weiterzuentwickeln.

Wie dieser Beitrag aufzeigt, fungiert die Verwendung von Farb- und Pflanzenmetaphorik als eine Art Spiegel der Adelsgesellschaft. In der Auswahl von Pflanzen, in der Gestaltung der Parkanlagen und auch in der Farbwahl spiegelt sich die Innenwelt Keyserlings Adliger wider. Die Künstlichkeit der Parkanlagen 
focus on German Studies. https://journals.uc.edu/index.php/fogs (ISSN 1076-5697) focus

on German Studies

innerhalb der Adelsenklave bildet eine Parallele zum Verhalten der Adligen: Ihr Leben ist geprägt von Konventionen, von Autorität und Strenge, gleichzeitig aber auch von Müßiggang, Unaufrichtigkeit und der Sehnsucht nach freien Liebesbeziehungen, die ihnen verwehrt bleiben. Innerhalb der Enklave haben

Keyserlings Adlige die (Schein-)Sicherheit, die sie im Außenraum nicht sehen. Die gesamt-gesellschaftlichen Umbrüche, die dafür sorgen, dass sich auch die Strukturen der Adelsgesellschaft verändern, verlangten vom realhistorischen Adel ein Umdenken. Keyserlings Beschreibungen des Adels sind nicht despektierlich und die Adligen werden nicht offenkundig kritisiert. Vielmehr scheint es so zu sein, dass Keyserling in seinen Schlossgeschichten ein Panorama der Adelsgesellschaft zeichnet, in dem sich der Untergang des Adels manifestiert. Im direkten Vergleich der Figuren zeigt sich jedoch auch eine Perspektive für die Adelsgesellschaft, die die jüngste Generation betrifft. Oda ist ein Beispiel dafür, dass es nicht der zwangsläufige Weg für die Adligen sein muss, wie eine Frucht in der Adelsenklave zu verfaulen. Das Aufbrechen alter Strukturen erscheint als Möglichkeit, sich als Adelsgemeinschaft neu zu definieren - etwas, das dem realhistorischen Adel nicht gelungen zu sein scheint.

\section{$\underline{\text { Literaturverzeichnis }}$}

Buchlaub, Sabine und Wefel, Claudia. „Die Landschaftsdarstellung in Eduard von Keyserlings Erzählwerk. Impressionismus - Romantik - Dekadenz“. Dekadenz in Deutschland. Beiträge zur Erforschung der Romanliteratur um die Jabrhundertwende, hrsg. von Dieter Kafitz. Lang, 1987, S. 243-257.

Birkhan, Helmut. „Pflanzen im Mittelalter. Eine Kulturgeschichte“. Böhlau Wien, 2013.

Conze, Eckart. „Von deutschem Adel: Die Grafen von Bernstorff im zwanzigsten Jahrhundert“. Deutsche Verlags-Anstalt, 2000.

Keyserling, Eduard von. „Schwüle Tage. Novelle“. Vollständige Ausgabe 1998, 7. Aufl. dtv 
focus on German Studies.

https://journals.uc.edu/index.php/fogs

Verlagsgesellschaft, 2018.

--- „Harmonie“. Eduard von Keyserling: Landpartie. Gesammelte Eræä̈hlungen hrsg. von Horst

Lauinger. Manesse Verlag, 2018, S. 104-144.

--- „Am Südhang““. Eduard von Keyserling: Landpartie. Gesammelte Eræähblungen hrsg. von Horst Lauinger. Manesse Verlag, 2018, S. 393-462.

Kimmich, Dorothee und Wilke, Tobias. „Einführung in die Literatur der Jahrhundertwende“.

Einführungen Germanistik. (2. aktualisierte Auflage). Wissenschaftliche Buchgesellschaft, 2011.

Lauinger, Horst. „Kommentar und Anhang“. Eduard von Keyserling: Landpartie. Gesammelte Errähblungen hrsg. von Horst Lauinger. Manesse Verlag, 2018, S. 655-740.

Markewitz, Sandra. „Ein letzter Impressionist - Eduard von Keyserling und die Farben“. Aisthesis Verlag, 2010.

--- „Natur, Kultur, Moderne - Philosophische Aspekte im Werk Eduard von Keyserlings“. Eduard von Keyserling und die Klassische Moderne hrsg. von Christoph Jürgensen und Michael Scheffel. J.B. Metzler, 2020, S. 247-263.

Schneider, Jens Ole. „Spätadlige Skepsis - Zur Modernereflexion in Keyserlings Erzählung Am Südhang (1911)“. Eduard von Keyserling und die Klassische Moderne hrsg. von Christoph Jürgensen und Michael Scheffel. J.B. Metzler, 2020, S. 147-166.

Schulz, Angela. „Ästhetische Existenz im Erzählwerk Eduards von Keyserling“. Lang, 1991.

Schwalb, Irmelin. „Eduard von Keyserling. Konstanten und Varianten in seinem erzählerischen Werk ab 1903“. Lang, 1993.

Weinhold, Ulrike. „Künstlichkeit und Kunst in der deutschsprachigen Dekadenz-Literatur“. Europäische Hochschulschriften; Reihe 1, Deutsche Literatur und Germanistik; 215. Lang, 1977. 
focus on German Studies.

https://journals.uc.edu/index.php/fogs

(ISSN 1076-5697)

\section{focus on German Studies}

Wünsch, Marianne. „Zur Ethnographie und den Geschlechterrollen im Erzählwerk Keyserlings“. Eduard von Keyserling und die Klassische Moderne hrsg. von Christoph Jürgensen und Michael Scheffel. J.B. Metzler, 2020, S. 203-214. 